\title{
Retinopathy of Prematurity: A Clinical Approach
}

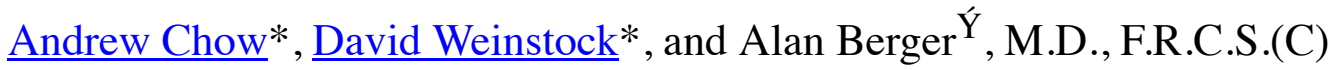 \\ * To whom correspondence should be addressed: Undergraduate Education Office, University of Toronto \\ Room 3245, 1-Kings College Circle, Toronto, ON, Canada M5S 1A8 \\ Ý \\ Department of Ophthalmology, University of Toronto
}

\section{INTRODUCTION}

Retinopathy of prematurity (ROP), previously known as retrolental fibroplasia, was first described by Terry in 1942 (1). It is a vasoproliferative disorder that is a leading cause of childhood blindness in the United States, causing 550 new cases of infant blindness each year (2). It occurs principally in premature infants treated with high concentrations of oxygen. There are two phases of ROP: (i) an acute phase in which normal vasculogenesis is interrupted, and (ii) a chronic phase in which vascular membranes proliferate into the vitreous. This proliferation can lead to retinal detachment, scarring of the macula, and significant visual loss (3).

This article summarizes the incidence, pathophysiology, and classification of ROP. Current protocols for evaluating and treating ROP, as well as long-term sequelae, are also described.

\section{INCIDENCE}

The overall incidence of ROP is $16-17 \%$ for all premature infants $(4,5)$. In infants with birth weight below 1251 grams, the incidence is 66\% (6). Major risk factors include: decreased gestational age, decreased birth weight, and supplemental oxygen therapy. Associated risk factors include acidosis, apnea, patent ductus arteriosus, septicemia, blood transfusions, and intraventricular hemorrhage (7).

\section{PATHOPHYSIOLOGY}

Normal blood vessels supplying the retina first appear at the beginning of the second trimester. Between the 16 th and 44th week of gestation, the vessels grow in a centrifugal pattern from the optic disc to the ora serrata (the far peripheral retina) to fully vascularize the retina (8). Consequently, the blood supply to the retina in an infant born prematurely will be incomplete. At the time of delivery, injury to the immature and developing retinal capillary bed, from oxygen or other etiological factors, can result in ROP. Once these capillaries are lost, they may begin to regrow following a variable delay period. Abnormal blood vessel growth may occur as a result of undetermined angiogenic factors. Eventually, the new vessel growth can either complete 
physiological vascularization of the retina or lead to the pathological changes associated with ROP, such as extra-retinal neovascularization, hemorrhages, scar formation, traction retinal folds, and retinal detachments (3).

\section{CLASSIFICATION OF CLINICAL MANIFESTATIONS}

In 1984, an international classification of ROP was established, and is based on three parameters: location, extent, and stage of the disease (9) (Table 1).

\section{Location}

The fundus is divided into three concentric zones centered on the optic disc (Fig. 1). Zone I extends in a circular fashion around the disc, with a radius defined as twice the disc-fovea distance. Zone II extends peripherally from the edge of zone I, reaching the ora serrata on the nasal side and the area of the anatomical equator on the temporal side. Zone III is the residual retina anterior to the edge of zone II temporally.

\section{Extent}

The extent of the disease is quantified as number of clock-hours of involvement. Therefore, as the examiner looks at the patient's eyes, pathology at the three o'clock position corresponds to the nasal side of the right eye and the temporal side of the left eye.

\section{Staging}

There are five stages of ROP. In stage 1, a thin demarcation line exists between the vascularized and avascular retina. Stage 2 is characterized by the demarcation line extending out of the plane of the retina to form a ridge. In stage 3 , in addition to the ridge, there is extra-retinal proliferation of vascular tissue extending into the vitreous. Stage 4 is characterized by partial retinal detachment. In addition, stage 4 can be further divided into stage 4A, which includes extra-foveal detachment, and stage 4B, where retinal detachment includes the fovea. Finally, stage 5 corresponds to total retinal detachment, which is always funnel-shaped. Either end of the funnel can be open or narrow (10).

A "plus" sign is added to the above stages if any of the following findings is present, and is regarded as an ominous prognostic indicator: dilatation and tortuosity of the posterior vessels, dilatation of iris vessels, rigid and poorly dilating pupil, and vitreous haze.

\section{DIAGNOSIS}

There exists a variety of screening protocols for the diagnosis of ROP. The American Academy of Pediatrics and American College of Obstetricians and Gynecologists recently recommended the following screening protocol for ROP in high risk infants (Table 2) (11). Briefly, it attempts to screen premature, low birthweight infants by initial fundoscopic examination at about six weeks of age. Follow-up eye examination is necessary to monitor the progress of retinal vascularization. The sensitivity of this protocol has been shown to be high such that the fewest number of severe cases of ROP are missed, while a high percentage of overall cases are detected (12).

Fundoscopy, performed by an ophthalmologist, is an essential component of the complete ophthalmologic examination. Examinations should occur in an intensive care unit setting with the assistance of a nurse. This examination makes use of cycloplegic and mydriatic drops, a topical anesthetic, an infant lid speculum, an indirect ophthalmoscope, a 20-diopter lens, and a scleral depressor. Commonly used agents include Cyclomydril (a combination of Cyclogyl 0.2\% and phenylephrine 1.0\%), and topical proparacaine 
hydrochloride $0.5 \%$, used as the anesthetic agent. Indirect ophthalmoscopy with scleral depression is required to view the peripheral fundus.

\section{TREATMENT}

Despite the extensive literature on the management of ROP, there are no generally agreed-upon guidelines for its treatment. Studies have focused on two areas: drug therapy, such as vitamin E supplementation, and surgical management (cryotherapy, laser photocoagulation, and vitrectomy), for the treatment of acute disease and the management of retinal detachment (13).

\section{Drug Therapy}

Anti-oxidant therapy has been proposed to scavenge free radicals that are potentially damaging to developing retinal vessels (14). Vitamin $\mathrm{E}$ has been tested in the prevention of ROP in premature infants with low birthweight. In several controlled studies, the prophylactic use of vitamin E did not affect the incidence of ROP $(15,16)$. In subsequent controlled trials, the use of higher doses of vitamin E significantly decreased the incidence of ROP in the treatment group. However, these trials also detected an increased incidence of septicemia and necrotizing enterocolitis in the treated group (17). Since most cases of ROP are mild in degree and regress completely without medical intervention, the risk/benefit ratio of pharmacological prophylaxis is unfavorable given the undesirable side effects (18). Furthermore, several prospective studies on vitamin E treatment for ROP have shown conflicting results (19-21). Therefore, at the present time, the literature does not conclusively indicate the use of vitamin $\mathrm{E}$ for the management of ROP.

\section{Surgical Management}

\section{Cryotherapy}

Cryotherapy refers to applications of cold lesions to the avascular retina anterior to the region of active disease. The Multicenter Trial of Cryotherapy for ROP (Cryo-ROP) $(22,23)$ defines "threshold ROP" as eyes that reach Stage 3+ in zone 1 or 2 over at least five contiguous or eight cumulative hours. In this randomized control trial, transscleral cryotherapy to the avascular retina was applied to only one eye when there was threshold retinopathy in both eyes, or in one-half of an affected eye when threshold severity was unilateral. The outcome was defined as unfavorable if there was (i) posterior retinal folding, which usually involved the macula, (ii) retinal detachment involving zone 1 of the posterior pole, or (iii) retrolental tissue or "mass" obscuring the view of the posterior pole. The results showed that an unfavorable outcome was significantly less frequent $(p<0.001)$ in the eyes that received cryotherapy $(31.1 \%)$ compared to untreated eyes $(51.4 \%)$ $(12,13)$. These findings therefore suggest that cryotherapy may be recommended for the treatment of a single eye in cases of bilateral threshold ROP. However, the decision to treat the other eye or to treat a single eye in unilateral threshold ROP should be judged individually. No conclusive recommendations are given for cryotherapy in patients with less severe disease. Close monitoring should be provided.

\section{Laser Photocoagulation}

Recently, laser photocoagulation has been proposed as an effective alternative to cryotherapy. This treatment consists of applying a laser beam delivered through an indirect ophthalmoscope. The laser light is absorbed by tissue pigments and is converted into heat. This thermal energy denatures cellular components and causes tissue coagulation. Preliminary results suggest that the effectiveness of laser therapy is comparable to that of cryotherapy in the treatment of ROP (24). Moreover, in laser photocoagulation, the retina is ablated by the laser beam directly. This is in contrast to cryotherapy which involves freezing the sclera, choroid, and retina. Consequently, fewer adverse effects are expected following the laser therapy (25). A recent study has shown that the incidence of intraocular hemorrhage is lower following laser therapy (15\%), compared with 
cryotherapy (22.3\%) (26). Additional advantages of laser therapy include ease of treatment, lower incidence of pain, and more discrete, less confluent scars (26). Additional studies are still required to establish the longterm consequences of laser therapy.

\section{Scleral Buckling and Vitrectomy}

While cryotherapy has proven beneficial in the treatment of stage 3 ROP, a considerable number of these cases still progress to stages 4 and 5. Partial retinal detachment in stage 4 ROP can sometimes be repaired with an encircling scleral buckle with or without drainage of subretinal fluid. Total retinal detachment in stage $5 \mathrm{ROP}$ requires vitrectomy for repair. According to recent studies, the anatomical attachment rate in stages $4 \mathrm{~B}$ and 5 varies between $28 \%$ (27) and $45 \%$ (28). However, visual prognosis after anatomic reattachment of the retina is poor. Between $4 \%$ (29) and 27\% (28) of eyes treated for ROP stage 5 have achieved a visual result of fixation and following

\section{SEQUELAE}

The most common sequelae of regressed ROP include poor visual acuity and a higher incidence of strabismus (especially esotropia), amblyopia myopia, and late retinal detachment (30). Consequently, it is of prime importance that such infants with regressed ROP receive long-term follow-up care by an ophthalmologist.

In contrast, progressive ROP can lead to legal blindness, closed-angle glaucoma, phthisis bulbi (shrunken globe), and entropion (25). A recent study demonstrated that 33\% of infants with advanced ROP had less than $20 / 200$ vision in their preferred eye (31). Moreover, the incidence of strabismus ranged from $23-47 \%$ in infants with ROP compared with 10-20\% in premature infants without ROP (32). Approximately one-half of ROP patients with strabismus are amblyopic $(31,33)$. In addition, between $10-15 \%$ of ROP children will develop cicatricial retinal changes (34). Among these patients, $80 \%$ may develop myopia (35) while $80 \%$ may develop seizures (31).

\section{DISCUSSION}

Retinopathy of prematurity (ROP) is a vasoproliferative disorder that is common among premature and low birth-weight infants, especially those treated with oxygen (6). Recently, the burden of ROP has been increasing due to a higher proportion of smaller infants surviving the neonatal period. Consequently, regular ophthalmologic examinations begun in the intensive care unit are increasingly important in order to identify infants with ROP. Appropriate treatment of ROP can reduce visual impairment. Modalities of therapy include vitamin $\mathrm{E}$ and other anti-oxidants, cryotherapy, laser photocoagulation, scleral buckling, and vitrectomy. However, cryotherapy is the only treatment that has been proven, in a well-designed randomized controlled trial (Cryo-ROP), to improve visual outcomes in bilateral threshold ROP.

Further research and clinical trials are needed to improve the understanding of ROP and to develop more

INTERNET ARCHIVE

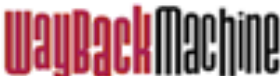



http://www.medicine.mcgill.ca/mjm/issues/v01n01/retino.html

16 captures

27 Jul $2003-28$ Oct 2012

effective strategies for the prevention and treatment of ROP.

\section{REFERENCES}


1. Terry TL. Extreme prematurity and fibroplastic overgrowth of persistent vascular sheath behind each crystalline lens. American Journal of Ophthalmology 1942:25:203-204.

2. Vaughan D, Asbury T, Riordan-Eva P. General Ophthalmology. Connecticut: Appleton and Lange, 1993:201.

3. Taylor D, et al. Pediatric Ophthalmology. Boston: Blackwell Scientific Publication, 1990:371-372.

4. Kaline RE. Retrolental fibroplasia - experience over two decades in one institution. Ophthalmology 1982;89:91-95.

5. Tasman W. The natural history of active retinopathy of prematurity. Ophthalmology 1984;91:1499-1502.

6. Palmer EA, Flynn JT, Hardy RJ, et al. Incidence and early course of retinopathy of prematurity. Ophthalmology 1991;98:1628-1640.

7. Flynn JT. Retinopathy of prematurity. Pediatric Clinics of North America 1987;34:1487-1516.

8. Weakley DR, Spencer R. Current concepts in retinopathy of prematurity. Early Human Development 1992;30:121-138.

9. The Committee for the Classification of Retinopathy of Prematurity. An international classification of Retinopathy of Prematurity. Archives of Ophthalmology 1984;102:1130-1134.

10. The International Committee for the Classification of the Late Stages of Retinopathy of Prematurity. An international classification of retinopathy of prematurity. Archives of Ophthalmology 1987;105:906-912.

11. American Academy of Pediatrics and American College of Obstetricians and Gynecologists. Guidelines for Perinatal Care, 3rd ed., Washington, DC, 1992.

12. Palmer EA. Optimal timing of examination for acute retrolental fibroplasia. Ophthalmology 1981;88:662668.

13. Taylor D, et al. Pediatric Ophthalmology. Boston: Blackwell Scientific Publication, Boston, 1990:372373.

14. Quinn GE, Johnson L, Otis CH, et al. Incidence, severity and time course of ROP in a randomized clinical trial of vitamin E prophylaxis. Documenta Ophthalmologica 1990;74:223-228.

15. Phelps DL, Rosenbaum AL, Isenberg SL, et al. Tocopherol efficacy and safety for preventing retinopathy of prematurity: a randomized, controlled, double-masked trial. Pediatrics 1987;79:489-500.

16. Schaffer DB, Johnson L, Quinn GE, et al. Vitamin E and retinopathy of prematurity. Follow up at 1 year. Ophthalmology 1985;92:1005-1011.

17. Johnson L, Bowen F, Hermann N. The relationship of prolonged elevation of serum vitamin E levels to neonatal bacterial sepsis and necrotizing enterocolitis. Pediatric Research 1983;17:319A.

18. Johnson L, Quinn GE, Abbasi S, et al. Effect of sustained vitamin E levels on incidence and severity of retinopathy of prematurity: a controlled clinical trial. Journal of Pediatrics 1989;114:827-838.

19. Finer NN, Schindler RF, Grant G, et al. Effect of intramuscular vitamin E on frequency and severity of 
retrolental fibroplasia. A controlled trial. Lancet 1982;1:1087-1091.

20. Pucklin JE, Simon RM, Ehrenkranz RA. Influence on retrolental fibroplasia of intramuscular vitamin E administration during respiratory distress syndrome. Ophthalmology 1982;89:96-103.

21. Ben Sira I, Nissenkorn I, Kremer I. Retinopathy of Prematurity. Survey of Ophthalmology 1988;33(1):116.

22. Cryotherapy for Retinopathy of Prematurity Cooperative Group. Multicenter trial of cryotherapy for retinopathy of prematurity. 3-month outcome. Cryotherapy for retinopathy of prematurity. Archives of Ophthalmology 1990;108:195-204.

23. Cryotherapy for Retinopathy of Prematurity Cooperative Group. Multicenter trial of cryotherapy for retinopathy of prematurity: 1-year outcome. Structure and function. Archives of Ophthalmology 1990;108:1408-1416.

24. McNamara JA, Tasman W, Brown GC, et al. Laser photocoagulation for stage 3+ retinopathy of prematurity. Ophthalmology 1991;98:576-580.

25. Phelps DL. Retinopathy of prematurity. Pediatric Clinics of North America 1993;40(4):705-714.

26. Flynn JT, Tasman W. Retinopathy of Prematurity - A clinician's guide. New York: Springer-Verlag, New York, 1992:63-71.

27. Quinn GE, Dobson V, Barr CH, et al. Visual acuity in infants after vitrectomy for severe retinopathy of prematurity. Ophthalmology 1991;98:5-13.

28. Trese MT. Surgical results of stage V retrolental fibroplasia and timing of surgical repair. Ophthalmology 1984;91:461-466.

29. Tasman W, Borrone RN, Bolling J. Open sky vitrectomy for total retinal detachment in retinopathy of prematurity. Ophthalmology 1987;94:449-452.

30. Albert DM, Jakobiec FA. Principles and practice of ophthalmology. Philadelphia: WB Saunder Co. Philadelphia, 1994:2809-2812.

31. Biglan AW, Cheng KP, Brown DR. Update on ROP. International Ophthalmology Clinics 1989;29:2-9.

32. Cats BP, Tan K. Premature with and without regressed ROP: comparison of long-term ophthalmological morbidity. Journal of Pediatric Ophthalmology 1989;26:271-275.

33. Snir M, Nissenkorn I, Sherf I, et al. Visual acuity, strabismus, amblyopia in premature babies with and without ROP. Annals of Ophthalmology 1988;20:256-258.

34. Kitchen WH, Yu VY, Orgill A, et al. Collaborative study of very low birth weight infants: outcome of 2year-old survivors. Lancet 1982;1:1457-1460.

35. Tasman W. Late complications of retrolental fibroplasia. Ophthalmology 1979;86:1724-1740. 
Andrew Chow and David Weinstock are presently third-year medical students at the University of Toronto (Toronto, Ontario). Their research on retinopathy of prematurity for this review article was conducted during their third year of medical education at the Department of Ophthalmology, University of Toronto. Their research and future specialty interests are in the field of ophthalmology. 